\title{
Barotrauma en COVID-19: Asociación de neumotórax y uso de cánula nasal de alto flujo
}

\section{Barotrauma in COVID-19: Association between pneumothorax and high flow nasal cannula use}

\author{
Felipe Navarrete' ${ }^{1}$, Allan Mix ${ }^{1}$
}

\section{Resumen}

La cánula nasal de alto flujo (CNAF) es una alternativa en la oxigenoterapia de pacientes en insuficiencia respiratoria aguda hipoxémica, especialmente en contexto de pandemia por SARS-CoV-2. Existen muchos beneficios, con distintos niveles de evidencia y muy pocos efectos adversos reportados a su uso descritos en adultos. Se presenta el caso de un paciente con enfermedad pulmonar obstructiva crónica, que cursando con una neumonía grave por SARS-CoV-2, mientras recibía terapia con CNAF, desarrolló un neumotórax. Esta complicación asociada a la terapia con CNAF es poco reportada en la literatura, lo que motiva este reporte.

Palabras clave: cánula Nasal de alto Flujo; barotrauma; neumotórax; SARS-CoV-2.

\begin{abstract}
High Flow Nasal Cannula (HFNC) is an alternative to deliver oxygen in patients with hypoxemic acute respiratory failure, especially in the context of SARS-CoV-2 outbreak. There are plenty of benefits described, with different evidence levels and very few adverse effects published in adults until now. We report the case of a patient with chronic obstructive pulmonary disease, presenting with SARS-CoV-2 severe pneumonia that while receiving oxygen with HFNC developed pneumothorax. This complication associated with HFNC has not been commonly described, which motivates this report.
\end{abstract}

Keywords: high Flow Nasal Cannula: Barotrauma; Pneumothorax; SARS-CoV-2.

Fecha de envío: 2020-11-30 - Fecha de aceptación: 2021-03-16

\section{Introducción}

La pandemia del nuevo coronavirus SARS-CoV-2, presenta un desafío en relación a la oxigenoterapia como manejo de la falla respiratoria aguda hipoxémica, que en muchos casos requiere ventilación mecánica invasiva (Spoletini et al., 2015). En este contexto, como alternativa o puente hacia esta, surge la cánula nasal de alto flujo (CNAF), que puede entregar hasta una $\mathrm{FiO}_{2}$ al $100 \%$ a un máximo de $60 \mathrm{~L} / \mathrm{min}$, calefaccionado y humidificado, derivando en una serie de efectos fisiológicos a su favor (Vargas et al., 2015), entre los que destaca la presión positiva al final de la espiración (PEEP) (Sztrymf et al, 2012). Aun así, el uso de este dispositivo no está exento de complicaciones, las cuales son infrecuentes y poco descritas en la literatura en adultos.

A continuación, se expone el caso de un paciente con neumonía por SARS-CoV-2, que presenta neumotórax asociado al uso de CNAF.

\section{Desarrollo de caso}

Paciente de 74 años, con antecedentes de hipertensión arterial crónica y enfermedad pulmonar obstructiva crónica (EPOC) por tabaco, consultó en Unidad de Urgencia Adulto de Hospital Félix Bulnes por 7 días de tos productiva, mialgias y disnea progresiva hasta reposo, sin contacto SARS-CoV-2. Ingresó en regulares condiciones, vigil, febril, con frecuencia respiratoria (FR) de 33/minuto, saturación de $85 \%$ ambiental, uso de musculatura accesoria, murmullo pulmonar disminuido globalmente, crépitos difusos y escasas sibilancias. PCR SARS-CoV-2 positiva, tomografía computada (TC) de tórax compatible con neumopatía multifocal (Figura 1). Al laboratorio de ingreso parámetros inflamatorios elevados y $\mathrm{PaFi}=95$.

Se inició oxigenoterapia con máscara de no recirculación en pronación, broncodilatadores, corticoides en dosis de estrés y antibióticos de amplio espectro (suspendidos a posteriori). 
Evolucionó con mala tolerancia a prono y sin cambios clínicos, por lo que se escaló a CNAF con $\mathrm{FiO}_{2}$ al $90 \%$ a $60 \mathrm{~L} / \mathrm{min}$, manteniendo disnea, signos clínicos de aumentado trabajo ventilatorio y PaFi en 55. Conversado con paciente y familia se decide limitar la terapia, excluyendo la intubación. Se implementa apoyo con ventilación mecánica no invasiva (VMNI) a CPAP 12 y $\mathrm{FiO}_{2}$ al 45\%, por 72 horas, con buen acople, mejoría de mecánica ventilatoria, PaFi de 147 y ventanas bien toleradas. Ante mejoría parcial clínica se reinstala CNAF a $60 \mathrm{~L} / \mathrm{min}$ con $\mathrm{FiO}_{2}$ al 90\%; manteniendo respuesta.

A las 24 horas posteriores a la reinstalación de CNAF, manteniendo parámetros previos, el paciente presentó dolor de inicio súbito en hemitórax derecho, con empeoramiento de mecánica ventilatoria, murmullo pulmonar abolido a derecha (no descrito previamente) y caída en saturación de $\mathrm{O}_{2}$. Se realizó radiografía de tórax, que evidenció neumotórax de $25 \%$ de campo pulmonar derecho, asociado a enfisema subcutáneo ipsilateral, por lo que se instaló pleurostomía, con reabsorción parcial de neumotórax al control (Figura 2). El paciente se mantuvo en malas condiciones, disneico a pesar de alto aporte de $\mathrm{O}_{2^{\prime}}$ con FR $>30$, desaturaciones hasta 76\%, PaFi de 40 y baja respuesta a analgesia. En este contexto, se optimizó sedoanalgesia. Horas después el paciente falleció.

\section{Figuras}

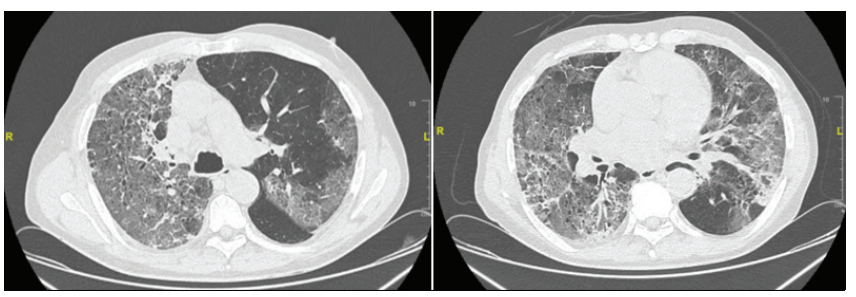

Figura 1: TC de tórax sin contraste en que se observan abundantes opacidades en vidrio esmerilado de predominio periférico y subpleurales difusas, mayores a derecha. No se observa neumotórax.

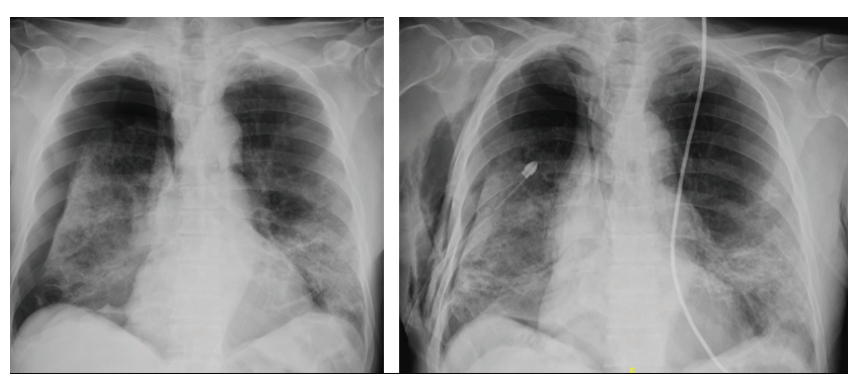

Figura 2: Radiografía de tórax posterior a inicio de dolor agudo. A. Neumotórax derecho. B. Tubo pleural in situ, con reabsorción parcial de neumotórax, se observa además enfisema subcutáneo no presente en estudio previo.

\section{Discusión}

Se ha demostrado que la CNAF puede aportar PEEP, disminuye la resistencia de la vía aérea, mejora el trabajo respiratorio, barre $\mathrm{CO} 2$, es relativamente cómoda, tiene un sistema poco complejo y un menor riesgo de aerosolización de partículas que VMNI (Vargas et al., 2015; Bruni et al., 2019). También se ha asociado su uso a disminución de la tasa de necesidad de intubación con respecto a oxigenoterapia convencional (Ni et al., 2017).

La suma de estos factores, ha hecho que durante la pandemia por SARS-CoV-2 se haya transformado en una alternativa terapéutica a VMNI, con resultados clínicos controversiales, beneficiosos en algunos reportes y series de casos ( Karamouzos et al., 2020; Rali et al., 2020), mostrando posibles reducciones en la necesidad de ventilación mecánica invasiva en pacientes cursando falla respiratoria hipoxémica moderada (Agarwal et al., 2020; Geng et al., 2020), aunque la mayoría de estos con calidad de evidencia baja (Bruni et al., 2019).

Por otro lado, hay evidencia de que su uso podría producir en algunos casos un retraso en el manejo invasivo de la vía aérea, con ello un aumento en la mortalidad (Kang et al., 2015) y otras complicaciones. Destaca entre ellas el barotrauma en forma de atrapamiento aéreo, neumotórax y neumomediastino; todas estas mayormente descritas en paciente crítico pediátrico (Shoemaker et al., 2007; Hegde \& Prodhan, 2013), razón por la que estos dispositivos incorporan válvulas de seguridad para evitar altas presiones, lo que no existe en los sistemas de CNAF para adultos, por ende, estas complicaciones son factibles en este grupo de pacientes (Nishimura, 2016). Este fenómeno ha sido descrito previamente; en un reporte de caso de adulto que posterior a extubación, se mantuvo 4 días con uso de CNAF, seguido a lo cual presentó neumomediastino, enfisema subcutáneo y neumotórax derecho, interpretado como secundario a la presión positiva entregada por la cánula posterior a la ruptura de una bula (Sonobe et al., 2019).

En esta misma línea, el caso en este reporte presenta a un paciente tabáquico, con EPOC, que en contexto de neumonía grave por SARS-CoV-2 previamente expuesto a VMNI, se instaló CNAF, evolucionando en 24 horas con neumotórax.

El tabaquismo y EPOC se asocian a aumento de riesgo de aparición de neumotórax secundario, especialmente en hombres, sin ninguna relación a función pulmonar basal (Hobbs et al., 2014). La terapia con VMNI si bien es una excelente alternativa, tiene como complicación ampliamente descrita al barotrauma, incluso existe evidencia que indica que los pacientes en ventilación mecánica cursando COVID-19 tienen aún más riesgo de este tipo de complicaciones (Edwads et al., 2020). 
Aun considerando estos factores, existe una relación temporal innegable al cambio de terapia a CNAF, pues a las 24 horas de iniciado este dispositivo, existe un quiebre clínico con nueva sintomatología que sugiere que se instala el neumotórax. Esto se explica por la capacidad de CNAF de otorgar una presión positiva al final de la espiración (PEEP), el cual se ha estimado un aumento de 3-5 cm de $\mathrm{H} 2 \mathrm{O}$ a flujos de 30-50L/min (Parke \& McGuinness, 2013), incluso se ha descrito una relación lineal entre flujo y presión de vía aérea a boca cerrada (Parke et al., 2011).

El aumento de PEEP descrito en contexto del paciente con pulmón crónicamente dañado, cursando una infección aguda por SARSCoV-2, genera un ambiente ideal para la aparición de un fenómeno de ocupación pleural por aire.

Este reporte no invalida el aporte terapéutico de la CNAF, sin embargo, no es cuestionable que, en caso de existir más reportes en esta línea, la terapia con CNAF se pueda considerar como contraindicación relativa en los pacientes con los factores de riesgo mencionados por la posible aparición de lesiones por barotrauma.

\section{Contribuciones y reconocimientos: Fuentes de financiamiento}

No hubo conflictos de interés ni se recibió financiamiento de ningún tipo. El Dr. Felipe Navarrete realizó concepción y diseño, redacción de manuscrito, revisión bibliográfica, revisión crítica. Mientras que el Dr. Allan Mix efectuó la concepción y diseño, revisión crítica, aprobación de manuscrito.

\section{Referencias}

Agarwal A, Basmaji J, Muttalib F, Granton D, Chaudhuri D, Chetan D, Hu M, Fernando SM, Honarmand K, Bakaa L, Brar S, Rochwerg B, Adhikari NK, Lamontagne F, Murthy S, Hui DSC, Gomersall C, Mubareka S, Diaz J V, Burns KEA, Couban R, Ibrahim Q, Guyatt GH, \&Vandvik PO. (2020). High-flow nasal cannula for acute hypoxemic respiratory failure in patients with COVID-19: systematic reviews of effectiveness and its risks of aerosolization, dispersion, and infection transmission. Canadian Journal of Anesthesia. 67, 1217-1248.

Bruni A, Garofalo E, Cammarota G, Murabito P, Astuto M, Navalesi P, Luzza F, Abenavoli L, \& Longhini F. (2019). High Flow Through Nasal Cannula in Stable and Exacerbated Chronic Obstructive Pulmonary Disease Patients. Reviews on Recent Clinical Trials. 14, 247-260.

Edwards JA, Breitman I, Bienstock J, Badami A, Kovatch I, Dresner L, Schwartzman A. (2020). Pulmonary barotrauma in mechanically ventilated coronavirus disease 2019 patients: A case series. Annals of Medicine and Surgery. 28, 24-29.
Geng S, Mei Q, Zhu C, Yang T, Yang Y, Fang X, \& Pan A. (2020). High flow nasal cannula is a good treatment option for COVID-19. Heart \& Lung. 49, 444-445.

Hegde S, \& Prodhan P. (2013). Serious air leak syndrome complicating high-flow nasal cannula therapy: a report of 3 cases. Pediatrics. 131, 939-944.

Hobbs BD, Foreman MG, Bowler R, Jacobson F, Make BJ, Castaldi PJ, San José Estépar R, Silverman EK, Hersh CP. (2014). Pneumothorax risk factors in smokers with and without chronic obstructive pulmonary disease. Annals of the American Thoracic Society. 11, 1387-1394.

Kang BJ, Koh Y, Lim C-M, Huh JW, Baek S, Han M, Seo H-S, Suh HJ, Seo GJ, Kim EY, \& Hong S-B. (2015). Failure of high-flow nasal cannula therapy may delay intubation and increase mortality. Intensive Care Medicine. 41, 623-632.

Karamouzos V, Fligou F, Gogos C, \& Velissaris D. (2020). High flow nasal cannula oxygen therapy in adults with COVID-19 respiratory failure. A case report. Monaldi Archives for Chest Disease. 90.

Ni Y-N, Luo J, Yu H, Liu D, Ni Z, Cheng J, Liang B-M, \& Liang Z-A. (2017). Can High-flow Nasal Cannula Reduce the Rate of Endotracheal Intubation in Adult Patients With Acute Respiratory Failure Compared With Conventional Oxygen Therapy and Noninvasive Positive Pressure Ventilation?: A Systematic Review and Meta-analysis. Chest. 151, 764-775.

Nishimura M. (2016). High-flow nasal cannula oxygen therapy in adults: Physiological benefits, indication, clinical benefits, and adverse effects. Respiratory Care. 61, 529-541.

Parke RL, Eccleston ML, \& McGuinness SP. (2011). The effects of flow on airway pressure during nasal high-flow oxygen therapy. Respiratory Care. 56, 1151-1155.

Parke RL, \& McGuinness SP. (2013). Pressures delivered by nasal high flow oxygen during all phases of the respiratory cycle. Respiratory Care, 58, 1621-1624.

Rali AS, Nunna KR, Howard C, Herlihy JP, \& Guntupalli KK. (2020). High-flow Nasal Cannula Oxygenation Revisited in COVID-19. Cardiac Failure Review. 6.

Shoemaker MT, Pierce MR, Yoder BA, \& DiGeronimo RJ. (2007). High flow nasal cannula versus nasal CPAP for neonatal respiratory disease: a retrospective study. Journal of Perinatology. 27, 85-91. 
Sonobe S, Inoue S, Nishiwada T, Egawa J, \& Kawaguchi M. (2019). A case of subcutaneous emphysema/mediastinal emphysema during the use of humidified high-flow nasal cannula. Journal of Anesthesia Clinical Reports. 5, 85.

Spoletini G, Alotaibi M, Blasi F, \& Hill N. (2015). Heated humidified high-flow nasal oxygen in adults: Mechanisms of action and clinical implications. Chest. 148, 253-261.
Sztrymf B, Messika J, Mayot T, Lenglet H, Dreyfuss D, \& Ricard J-D. (2012). Impact of high-flow nasal cannula oxygen therapy on intensive care unit patients with acute respiratory failure: a prospective observational study. Journal of Critical Care. 27, 9-13.

Vargas F, Saint-Leger M, Boyer A, Bui NH, \& Hilbert G. (2015). Physiologic effects of high-flow nasal Cannula oxygen in critical care subjects. Respiratory Care. 60, 1369-1376. 